\title{
PERIODIC MOTION OF RIGID BODY IN CIRCULAR ORBIT
}

\author{
Z. NiEDZielsKa AND P. WĄż \\ Toruń Centre for Astronomy, N. Copernicus University \\ Piwnice, 87-148 Eysomice, Poland
}

(Received April 4, 1997)

\begin{abstract}
The periodic rotations of a symmetric rigid body close to the flat motions are analytically determined. Their orbital stability is investigated. Calculations are done up to the second order terms of a small parameter.

PACS numbers: 03.20.+i, 46.10.+z, 46.90.+s, 95.10.Ce
\end{abstract}

\section{Introduction}

Let us consider a rigid body whose mass center moves in a circular orbit around a point gravitational center. This problem was studied by many authors [1-4]. We assume that the body is symmetrical which allows us to reduce the equations of motion by one degree of freedom (projection of absolute angular velocity onto symmetry axis is constant of motion).

If moments of inertia are equal and projection of absolute angular velocity onto symmetry axis is equal to zero then there exist the so-called flat periodic rotations (symmetry axis lies in the orbital plane). Assuming that polar and equatorial moments of inertia are almost equal and projection of the angular velocity onto symmetry axis is small, Markeev [3] proved the existence of spatial periodic solutions close to the flat ones. Maciejewski and Niedzielska [5] adopted the method of Markeev to solve a similar problem for a rigid body whose mass center is located at the triangular libration point of the restricted three-body problem. They found that some results of Markeev [3] are incorrect and they presented a new, revised analysis. Authors of the above-mentioned papers only considered the particular family of periodic solutions - solutions with the same period as the generating motions. In this case basing on Poincaré's theory (Siegel and Moser [6]) they could state that under some assumptions there exists exactly one such family.

The purpose of our paper is to find the family of periodic solutions close to the flat rotations with a period close to the period of generating solution. We investigate periodic solutions lying on the same energy level surface as the generating motions. We also present the analysis of their orbital stability using the method of local analysis in the neighborhood of the periodic solution (Brjuno [7]). 


\section{Equations of motion and periodic solutions}

We assume that the center of mass $O_{1}$ of a rigid body moves in a circular orbit around the point gravitational center $O$. We choose the time unit in such a way that the orbital period is equal to one.

We assume that the orbit of the body lies in the $X Y$-plane of the fixed inertial reference frame $O X Y Z$ (see Fig. 1). In order to describe its rotational motion, we introduce two right handed orthonormal reference frames with origins at the mass center of the rigid body. The $x$-axis of the orbital frame is directed along the radius vector and the $z$-axis is parallel to the orbital angular momentum vector. The second frame is the principal axis reference frame.

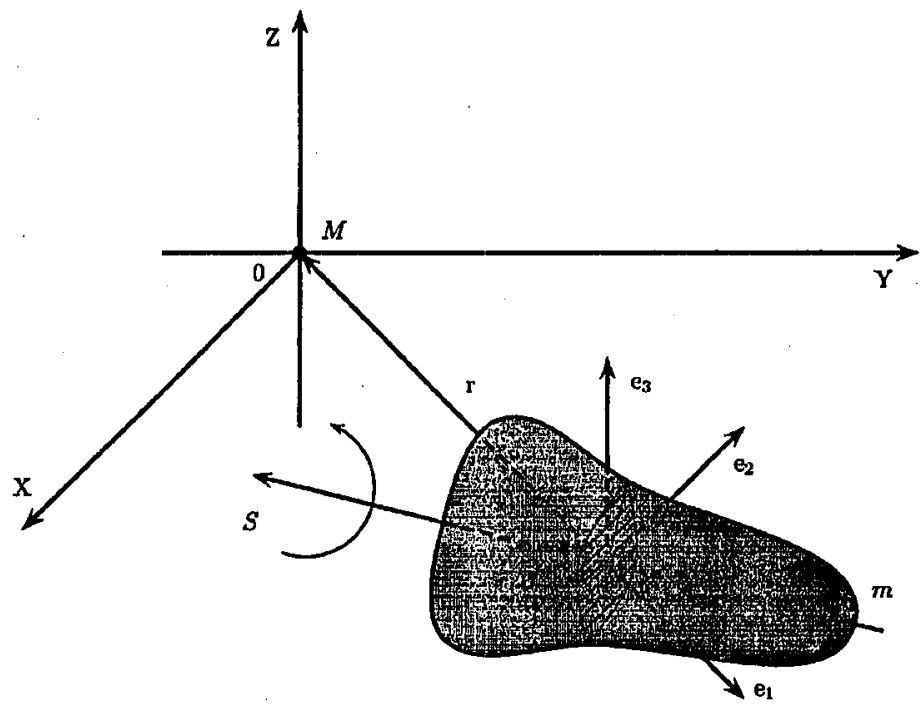

Fig. 1. A symmetric rigid body in a circular orbit. Geometry of the problem. $O X Y Z$ is the inertial reference frame. $e_{1}, e_{2}, e_{3}$ denote versors of the orbital frame. $S$ is the axis of the symmetry of the rigid body.

We parametrize the orientation of the principal reference frame with respect to the orbital reference frame by Euler angles $q_{1}, q_{2}, q_{3}$ of the type 3-2-1. Let the body possess an axis of dynamical symmetry. Without any loss of generality, we assume it is the first principal axis $(A, B, C$ are the principal moments of inertia and consequently $B=C$ ). The IIamiltonian of the problem can be written in the following form:

$$
H=\frac{1}{2}\left[\frac{p_{1}-p_{3} \sin \left(q_{2}\right)}{\cos \left(q_{2}\right)}\right]^{2}+\frac{1}{2} p_{2}^{2}-p_{1}+\frac{3}{4}(\alpha-1) \cos ^{2}\left(q_{2}\right)\left[1+\cos \left(2 q_{1}\right)\right]
$$

where $p_{1}, p_{2}, p_{3}$ are generalized momenta conjugated with $q_{1}, q_{2}, q_{3}$ and $\alpha=A / B$. According to (1) $q_{3}$ is a cyclic coordinate and $p_{3}$ is a constant of motion.

We assume that $A$ and $B$ are almost equal (the body is almost symmetric) 
and $p_{3}$ is small. Consequently, a small parameter $\mu$ can be introduced in the following manner:

$$
\begin{array}{ll}
\mu=(1-\alpha) / 2, \quad \mu \ll 1, \\
\gamma=p_{3}(0)=\mu \beta & \beta \sim 1 .
\end{array}
$$

The analysis was done up to the second order of the small values of $\mu$. Therefore the IIamiltonian function (1) takes the form

$$
H=H^{(0)}+\mu H^{(1)}+\mu^{2} H^{(2)}
$$

where

$$
\begin{aligned}
& H^{(0)}=\frac{p_{1}^{2}}{2 \cos ^{2}\left(q_{2}\right)}+\frac{p_{2}^{2}}{2}-p_{1}, \\
& H^{(1)}=\frac{\beta p_{1} \sin \left(q_{2}\right)}{\cos ^{2}\left(q_{2}\right)}+\frac{3}{2} \cos ^{2}\left(q_{2}\right)\left[1+\cos \left(2 q_{1}\right)\right], \\
& H^{(2)}=\beta^{2} \tan ^{2}\left(q_{2}\right) .
\end{aligned}
$$

For $\mu=0$ there exist flat $T_{0}$-periodic solutions of Hamilton's equation with Hamiltonian (3):

$$
p_{2}(t)=0, \quad q_{2}(t)=0, \quad p_{1}(t)=p_{1}(0)=\omega, \quad q_{1}(t)=\Omega t,
$$
where $\Omega=\omega-1, T_{0}=2 \pi /|\Omega|$.

The aim of this work is determination and analysis of rotational motion of the rigid body when $\mu \neq 0$ however is sufficiently small. Applying Poincaré's method (Siegel and Moser [6]) one can prove that if

$$
\frac{1}{\Omega} \neq m \quad(m \text { is an integer) }
$$

then for $\mu$ small enough there exists a $T$-periodic solution of Hamilton's equations with IIamiltonian (3). This solution lies on the same isoenergetic surface as the generating solution (4), it is analytic with respect to $\mu$ and it tends to the generating solution as $\mu \rightarrow 0$.

This solution as well as its period can be given explicitly as power series of $\mu$

$$
\begin{aligned}
q_{1}(t) & =\Omega t-\mu \frac{3[2 \Omega t+\sin (2 \Omega t)]}{4 \Omega^{2}}+\mu^{2}\left\{\frac{\beta^{2} t}{2 \Omega}-\frac{9 t \sin (\Omega t)}{64 \Omega^{4}}\right. \\
& \left.-\frac{9 t}{16 \Omega^{4}}[5 \Omega+4 \Omega \cos (2 \Omega t)+4 \sin (2 \Omega t)]\right\}+\ldots \\
q_{2}(t) & =-\mu \frac{\beta}{\omega}-3 \mu^{2} \beta\left\{\frac{-1-2 \Omega+3 \Omega^{2}}{2 \Omega\left(\Omega^{2}-1\right) \omega}-\frac{\cos (2 \Omega l)}{2 \Omega\left(\Omega^{2}-1\right)}\right\}+\cdots \\
p_{1}(t) & =\omega-3 \mu \frac{1+\cos (2 \Omega t)}{2 \Omega}+\mu^{2}\left\{\frac{-45+8 \beta^{2} \Omega^{2}}{16 \Omega^{3}}\right. \\
& \left.-\frac{9 \cos (2 \Omega t)-\cos (4 \Omega t)}{4 \Omega^{3}}-\frac{18 t \sin (2 \Omega t)}{4 \Omega^{2}}\right\}+\ldots \\
p_{2}(t) & =-\mu^{2} \frac{3 \beta}{\Omega^{2}-1} \sin (2 \Omega t)+\ldots \\
T= & \frac{2 \pi}{\Omega}+\mu \frac{3 \pi}{\Omega^{3}}+\mu^{2} \frac{\pi\left(81-8 \beta^{2} \Omega^{2}\right)}{8 \Omega^{5}}+\ldots
\end{aligned}
$$




\section{Orbital stability}

The periodic solution (6) is evidently unstable in the Lapunov sense. We will study its orbital stability using the method of normalization of the Hamiltonian in a neighborhood of the periodic solution. The algorithm proposed by Brjuno [7] consists of the following steps:

- introduction of the local coordinate system in a neighborhood of the periodic solution,

- introduction of new independent variable (instead of time),

- expansion of IIamiltonian function in a neighborhood of the periodic solution into the power series with respect to the coordinates,

- linear normalization and analysis of stability in linear approximation,

- nonlinear normalization and investigation of stability in resonant and nonresonant cases.

In order to determine orbital stability of the periodic solution (6) we made some canonical transformations. Since generating functions of these transformations have a complicated form, they are not present here. In order not to introduce too many symbols we denote new variables as primed but after transformation we will use unprimed symbols, again.

First, we transform our solution (6) by means of the canonical transformation

$$
\left(q_{1}, q_{2}, p_{1}, p_{2}\right) \rightarrow\left(\dot{W}_{1}, q_{2}^{\prime}, I_{1}, p_{2}^{\prime}\right)
$$

to the standard form. In the new coordinates the solution (6) takes the following form:

$$
W_{1}=\Omega t, \quad q_{2}=0, \quad I_{1}=0, \quad p_{2}=0
$$

and Hamiltonian can be written as

$$
H=\sum_{l=2}^{\infty} H_{l},
$$

where $H_{l}$ is a homogeneous polynomial of order $l$ with respect to variables $\sqrt{I_{1}}, q_{2}, p_{2}$ and its coefficients are analytic with respect to $\mu$

$$
H_{l}=H_{l}^{(0)}+\mu H_{l}^{(1)}+\mu^{2} H_{l}^{(2)}+\mu^{3} H_{l}^{(3)} \ldots
$$

The terms of the second and third order, necessary for the further analysis, are given below

$$
\begin{aligned}
& H_{2}^{(0)}=\frac{1}{2}|\omega|\left(q_{2}^{2}+p_{2}^{2}\right)+\Omega I_{1} \\
& H_{2}^{(1)}=\frac{-3}{2 \Omega} I_{1}-\frac{3(1+2 \Omega)\left[1+\cos \left(2 W_{1}\right)\right]}{2 \Omega|\omega|} q_{2}^{2},
\end{aligned}
$$




$$
\begin{aligned}
H_{2}^{(2)} & =\frac{-45+8 \beta^{2} \Omega^{2}}{16 \Omega^{3}} I_{1}+\frac{\beta^{2}(\omega+\Omega)}{2 \Omega|\omega|} q_{2}^{2}-\frac{9 \cos \left(2 W_{1}\right)}{4 \Omega^{3}|\omega|} q_{2}^{2} \\
& -\frac{9\left\{\left(2+\left[3-\cos \left(4 W_{1}\right)\right]+4 \Omega\left[1-\cos \left(4 W_{1}\right)\right]\right\}\right.}{16 \Omega^{3}|\omega|} q_{2}^{2}, \\
H_{3}^{(0)} & =0, \\
H_{3}^{(1)} & =\frac{-\beta}{\sqrt{|\omega|}} q_{2} I_{1}+\frac{-\beta \omega}{2|\omega| \sqrt{|\omega|}} q^{3}, \\
H_{3}^{(2)} & =\frac{3 \beta \sqrt{|\omega|} \sin \left(2 W_{1}\right)}{\Omega\left(\Omega^{2}-1\right)} I_{1} p_{2}-\frac{12 \beta \Omega^{2}+3 \beta \omega^{2} \cos \left(2 W_{1}\right)}{2 \Omega^{2} \sqrt{|\omega|} \omega^{2}} q_{2} I_{1} \\
& +\frac{3 \beta \sin \left(2 W_{1}\right)}{\Omega \sqrt{|\omega|}(\Omega-1)} q_{2}^{2} p_{2}-\frac{3 \beta[-1+\Omega(8-7 \Omega)]}{4 \Omega|\omega| \sqrt{|\omega|}\left(\Omega^{2}-1\right)} q_{2}^{3} \\
& -\frac{\cos \left(2 W_{1}\right)\left(3+13 \Omega^{2}\right)}{4 \Omega|\omega| \sqrt{|\omega|}\left(\Omega^{2}-1\right)} q_{2}^{3}, \\
H_{4}^{(0)} & =\frac{1}{2} I_{1}^{2}+\frac{\omega}{|\omega|} I_{1} q_{2}^{2}+\frac{1}{3} q_{2}^{4} .
\end{aligned}
$$

At first we consider the stability of the periodic solution in the linear approximation. From the linear IIamilton's equations

$$
\begin{aligned}
& \dot{W}_{1}=\frac{\partial H_{2}}{\partial I_{1}}=\Omega, \quad \dot{I}_{1}=-\frac{\partial H_{2}}{\partial W_{1}}, \\
& \dot{q}_{2}=\frac{\partial H_{2}}{\partial p_{2}}, \quad \dot{p}_{2}=-\frac{\partial H_{2}}{\partial q_{2}},
\end{aligned}
$$

we separate a subsystem for $q_{2}$ and $p_{2}$ with the independent variable $W_{1}$.

$$
\frac{\mathrm{d} q_{2}}{\mathrm{~d} W_{1}}=\frac{\partial F}{\partial p_{2}}, \quad \frac{\mathrm{d} p_{2}}{\mathrm{~d} W_{1}}=-\frac{\partial F}{\partial q_{2}},
$$

where Hamiltonian $F$ is $\pi$-periodic in $W_{1}$

$$
\begin{aligned}
F= & F_{0}+\mu F_{1}+\mu^{2} F_{2}+\ldots, \\
F_{0}= & \frac{|\omega|}{2 \Omega}\left(p_{2}^{2}+q_{2}^{2}\right), \quad F_{1}=\frac{3|\omega|}{4 \Omega^{3}}\left(p_{2}^{2}+q_{2}^{2}\right)-\frac{3(1+2 \Omega)}{2 \Omega^{2}|\omega|}\left[1+\cos \left(W_{1}\right)\right] q_{2}^{2}, \\
F_{2}= & \frac{9|\omega|}{8 \Omega^{5}}\left(p_{2}^{2}+q_{2}^{2}\right)-\frac{|\omega|}{32 \Omega^{5}}\left(-45+8 \beta^{2} \Omega^{2}\right)\left(p_{2}^{2}+q_{2}^{2}\right) \\
& -\mu^{2} \frac{9(1+2 \Omega)}{4 \Omega^{4}|\omega|}\left[1+\cos \left(2 W_{1}\right)\right] q_{2}^{2}+\frac{\beta^{2}(1+2 \Omega)}{2 \Omega^{2}} q_{2}^{2} \\
& -\frac{45+36 \Omega+36 \cos \left(2 W_{1}\right)-9 \cos \left(4 W_{1}\right)(1+4 \Omega)}{16 \Omega^{4}|\omega|} q_{2}^{2} .
\end{aligned}
$$


From the formal point of view in the system (17) instability may appear due to the parametric resonance (i.e. when $|\omega| / \Omega$ is an integer). However, in our case it is impossible because of condition (5). Thus, for small $\mu$ the periodic solution (6) is stable in linear approximation.

In order to study the influence of nonlinear terms we normalize $\mathrm{H}_{2}$. Then we introduce canonical variables $I_{2}$ and $W_{2}$ defined by

$$
q_{2}=\sqrt{2 I_{2}} \sin W_{2}, \quad p_{2}=\sqrt{2 I_{2}} \cos W_{2} .
$$

In the new variables the Hamiltonian (8) may be written in the following form:

$$
H=\sum_{l=2}^{\infty} H_{l},
$$

where

$$
\begin{aligned}
H_{2}= & \Omega_{\mu} I_{1}+\sigma \Omega_{\mu} I_{2}, \\
H_{3}= & I_{1} \sqrt{I_{2}}\left[A_{1} \sin W_{2}+A_{2} \sin \left(W_{2}-2 W_{1}\right)+A_{3} \sin \left(W_{2}+2 W_{1}\right)\right] \\
& +I_{2} \sqrt{I_{2}}\left[B_{1} \sin W_{2}+B_{2} \sin \left(3 W_{2}\right)+B_{3} \sin \left(3 W_{2}-2 W_{1}\right)\right. \\
& \left.+B_{4} \sin \left(3 W_{2}+2 W_{1}\right)+B_{5} \sin \left(W_{2}-2 W_{1}\right)+B_{6} \sin \left(W_{2}+2 W_{1}\right)\right], \\
H_{4}^{(0)} & =\frac{1}{2} I_{1}^{2}+\frac{\omega}{|\omega|} I_{1} I_{2}\left[1-\cos \left(2 W_{2}\right)\right]+\frac{1}{6} I_{2}^{2}\left[3-4 \cos \left(2 W_{2}\right)+\cos \left(4 W_{2}\right)\right] .
\end{aligned}
$$

In the formulae (21)-(23) $\sigma, A_{i}$ and $B_{i}$ are analytic functions of $\mu$ and

$$
\begin{aligned}
& \sigma=\sigma_{0}+\mu \sigma_{1}+\mu^{2} \sigma_{2}+\ldots \\
& A_{i}=\mu A_{i}^{(1)}+\mu^{2} A_{i}^{(2)}+\ldots, \quad i=1,2,3, \\
& B_{i}=\mu B_{i}^{(1)}+\mu^{2} B_{i}^{(2)}+\ldots, \quad i=1,2, \\
& B_{i}=\mu^{2} B_{i}^{(2)}+\ldots, \quad i=3, \ldots, 6 .
\end{aligned}
$$

The explicit forms of the coefficients $A_{i}, B_{i}$ are given in Appendix.

In order to investigate stability in nonlinear sense we have to consider the third and fourth order resonances. The resonance conditions can be written as

$n \sigma=2 k, \quad k$ is an integer,

where $n$ is the order of resonance.

From the form of the third order terms (22) one can state that the third order resonances may appear when

$$
3 \sigma=-2 \text { or } 3 \sigma=+2 \text {. }
$$

Since the equation $3 \sigma=2$ for small $\mu$ has no solution, only the first of the above possibilities may be fulfilled. Solving the equation $3 \sigma=-2$ we obtain two resonance curves

$$
\begin{aligned}
& \Omega=-\frac{3}{5}+\frac{9}{4} \mu+\text { terms of order } \mu^{2} \text { and higher, } \\
& \Omega=-3-\frac{9}{4} \mu+\text { terms of order } \mu^{2} \text { and higher. }
\end{aligned}
$$


The stability of our periodic solution in the third order resonance case depends on the value of the resonant terms (Markeev [8])

$$
B_{4} \sin \left(3 W_{2}+2 W_{1}\right) \text {. }
$$

If parameters $\Omega$ and $\mu$ lie on the curve (27) then $B_{4} \neq 0$ and the periodic solution is orbitally unstable. For parameters lying on the resonant curve (26) $B_{4} \sim \mu^{3}$. In order to determine the stability one should take into account terms of the order $\mu^{3}$.

If the third order resonances do not appear, IIamiltonian (20) can be transformed, using the canonical transformations to the following form:

$$
H=\Omega I_{1}+\sigma \Omega I_{2}+c_{20} I_{1}^{2}+c_{11} I_{1} I_{2}+c_{02} I_{2}^{2}+r I_{2}^{2} \cos \left(4 W_{2}+2 W_{1}\right)+\ldots,
$$

where

$$
\begin{aligned}
& c_{i j}=c_{i j}^{(0)}+\mu c_{i j}^{(1)}+\mu^{2} c_{i j}^{(2)}+\ldots, \\
& c_{20}^{(0)}=\frac{1}{2}, \quad c_{11}^{(0)}=\frac{\omega}{|\omega|}, \quad c_{02}^{(0)}=\frac{1}{2} .
\end{aligned}
$$

The coefficient $r$ is connected with the appearance of the fourth order resonances. If the fourth order resonance $4 \sigma=-2$ does not appear then $r=0$, in the opposite case $r \sim \mu$.

Now we apply Kolmogorov-Arnold-Moser (KAM) theory and its modification $[2,9]$ to the resonant case. Since the quantity

$$
\begin{aligned}
D & =c_{20}(\sigma \Omega)^{2}+c_{11} \Omega(\sigma \Omega)+c_{02} \Omega^{2} \\
& =\frac{1}{2} \Omega^{2}\left(\sigma_{0}-\frac{\omega}{|\omega|}\right)^{2}+\text { terms of order } \mu \text { and higher }
\end{aligned}
$$

is different from zero, the periodic solution is orbitally stable in the nonresonant case as well as in the fourth order resonance case.

\section{Conclusions}

The family of periodic solutions close to the flat rotations was found. The analysis of orbital stability for periodic solutions belonging to that family was done. We found that the determined periodic solutions are orbitally stable for all values of $\Omega$ (for which they exist) except for those values of $\mu$ and $\Omega$ lying on one branch of the third order resonance curve.

\section{Appendix}

Coefficients $A_{i}, B_{i}$ of the normal form (22)

$$
\begin{aligned}
& \Omega_{\mu}=\Omega+\mu \frac{-3}{2 \Omega}+\mu^{2} \frac{9-8 \beta^{2} \Omega^{2}}{16 \Omega^{3}}+\ldots, \\
& A_{1}^{(1)}=\beta \sqrt{\frac{2}{|\omega|}}, \\
& A_{1}^{(2)}=\frac{\sqrt{2} \beta\left(3+12 \Omega+15 \Omega^{2}+6 \Omega^{3}+24 \Omega \omega^{2}\right)}{4 \Omega \omega^{4} \sqrt{|\omega|}},
\end{aligned}
$$




$$
\begin{aligned}
& A_{2}^{(1)}=0 \\
& A_{2}^{(2)}=\frac{3 \sqrt{2} \beta(-1+\Omega-2|\omega|)(-\omega+2 \Omega|\omega|)}{8 \Omega^{2}\left(\Omega^{2}-1\right)(\Omega-|\omega|) \sqrt{|\omega|}} \\
& A_{3}^{(1)}=0 \\
& A_{3}^{(2)}=\frac{3 \sqrt{2} \beta(-1+\Omega+2|\omega|)(\omega+2 \Omega|\omega|)}{8 \Omega^{2}\left(\Omega^{2}-1\right)(\Omega+|\omega|) \sqrt{|\omega|}} \\
& B_{1}^{(1)}=\frac{-3 \sqrt{2} \beta \omega}{4|\omega| \sqrt{|\omega|}}, \\
& B_{1}^{(2)}=\frac{-3 \sqrt{2} \beta \mu^{2}}{16 \Omega \omega^{2} \sqrt{|\omega|}}\left[\frac{9\left(1+4 \Omega+5 \Omega^{2}+2 \Omega^{3}\right)}{\omega^{2}}+6(7 \Omega-1)\right] . \\
& B_{2}^{(1)}=\frac{\sqrt{2} \beta \omega}{4|\omega| \sqrt{|\omega|}}, \\
& B_{2}^{(2)}=\frac{\sqrt{2} \beta \mu^{2}}{16 \Omega \omega^{2} \sqrt{|\omega|}}\left[\frac{9\left(1+4 \Omega+5 \Omega^{2}+2 \Omega^{3}\right)}{\omega^{2}}+6(7 \Omega-1)\right] \\
& B_{3}^{(1)}=0,
\end{aligned}
$$$$
B_{3}^{(2)}=\frac{\sqrt{2} \beta}{\left(\Omega^{2}-1\right) \Omega\left(\Omega^{2}-\omega^{2}\right) \sqrt{|\omega|}}\left[\frac{9+15 \Omega-9 \Omega^{2}-15 \Omega^{3}+32 \Omega^{4}}{32|\omega|}\right.
$$$$
\left.+\frac{3\left(3+5 \Omega-5 \Omega^{2}+3 \Omega^{3}+10 \Omega^{4}\right)}{32 \Omega}-\frac{|\omega|\left(3+13 \Omega^{2}\right)}{16}-\frac{3 \omega^{3}}{4}\right],
$$$$
B_{4}^{(1)}=0
$$$$
B_{4}^{(2)}=\frac{\sqrt{2} \beta}{\left(\Omega^{2}-1\right) \Omega\left(\Omega^{2}-\omega^{2}\right) \sqrt{|\omega|}}\left[\frac{9+15 \Omega-9 \Omega^{2}-15 \Omega^{3}+32 \Omega^{4}}{32|\omega|}\right.
$$$$
\left.+\frac{3\left(-3-5 \Omega+5 \Omega^{2}-3 \Omega^{3}-10 \Omega^{4}\right)}{32 \Omega}-\frac{|\omega|\left(3+13 \Omega^{2}\right)}{16}+\frac{3 \omega^{3}}{4}\right],
$$

$$
B_{5}^{(1)}=0
$$




$$
\begin{aligned}
& B_{5}^{(2)}=\frac{-\sqrt{2} \beta}{\left(\Omega^{2}-1\right) \Omega\left(\Omega^{2}-\omega^{2}\right) \sqrt{|\omega|}}\left[\frac{9+15 \Omega-9 \Omega^{2}-15 \Omega^{3}+32 \Omega^{4}}{16|\omega|}-\frac{3 \omega^{3}}{4}\right. \\
& \left.+\frac{3\left(3+5 \Omega-5 \Omega^{2}+3 \Omega^{3}+10 \Omega^{4}\right)}{16 \Omega}+\frac{|\omega|\left(6+9 \Omega-6 \Omega^{2}-25 \Omega^{3}\right)}{8 \Omega}\right] \\
& B_{6}^{(1)}=0 \\
& B_{6}^{(2)}=\frac{-3 \sqrt{2} \beta}{\left(\Omega^{2}-1\right) \Omega\left(\Omega^{2}-\omega^{2}\right) \sqrt{|\omega|}}\left[\frac{9+15 \Omega-9 \Omega^{2}-15 \Omega^{3}+32 \Omega^{4}}{32|\omega|}\right. \\
& \left.+\frac{-3-5 \Omega+5 \Omega^{2}-3 \Omega^{3}-10 \Omega^{4}}{32 \Omega}+\frac{|\omega|\left(4+5 \Omega-4 \Omega^{2}-21 \Omega^{3}\right)}{16 \Omega}+\frac{\omega^{3}}{4}\right]
\end{aligned}
$$

\section{Reforences}

[1] V.V. Beletskii, Motion of the Salellite about its Mass Center in the Gravitational Field, Moscow University Press, Moscow 1975.

[2] A.P. Markeev, Kosm. Issled. 13, 322 (1975).

[3] A.P. Markecv, Koṣm. Issled. 23, 323 (1985).

[4] A. Maciejewski, Acla Astron. 44, 301 (1994).

[5] A. Maciejewski, Z. Niedzielska, Celest. Mech. Dyn. Astron. 49, 31 (1990).

[6] C.L. Siegel, J. Moser, Lectures on Celestial Mechanics, Springer-Verlag, Berlin 1971.

[7] A.D. Brjuno, Matemat. Sbor. 83, 273 (1970).

[8] A.P. Markeev, Libration Points in Celestial Mechanics and Kosmodynamics, Nauka, Moscow 1978 (in Russian).

[9] A.D. Brjuno, Usp. Mat. Nauk 43, 59 (1989). 